\title{
On the limit matrix obtained in the homogenization of an optimal control problem
}

\author{
S KESAVAN* and M RAJESH \\ ${ }^{*}$ The Institute of Mathematical Sciences, C.I.T. Campus, Taramani, Chennai 600113 , \\ India \\ Department of Mathematics, Indian Institute of Science, Bangalore 560 012, India \\ E-mail: kesh@imsc.ernet.in; rajesh@math.iisc.ernet.in \\ MS received 6 January 2001
}

\begin{abstract}
A new formulation for the limit matrix occurring in the cost functional of an optimal control problem on homogenization is obtained. It is used to obtain an upper bound for this matrix (in the sense of positive definite matrices).
\end{abstract}

Keywords. Homogenization; optimal control; elliptic equations.

\section{Introduction}

Let $\Omega \subset \mathbb{R}^{N}$ be a bounded domain. Let $0<\alpha_{m}<\alpha_{M}$. We denote by $\mathcal{M}\left(\alpha_{m}, \alpha_{M}, \Omega\right)$ the set of all $N \times N$ matrices $A=A(x)$, with coefficients in $L^{\infty}(\Omega)$, such that

$$
\alpha_{m}|\xi|^{2} \leq A(x) \xi \cdot \xi \leq \alpha_{M}|\xi|^{2} \text {, a.e. } x, \text { for all } \xi \in \mathbb{R}^{N} .
$$

Given a family of matrices $A_{\varepsilon} \in \mathcal{M}\left(\alpha_{m}, \alpha_{M}, \Omega\right)$, let $v_{\varepsilon} \in H_{0}^{1}(\Omega)$ be the unique (weak) solution of the problem

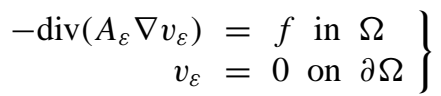

where $f \in L^{2}(\Omega)$ is given. Then $\left\{v_{\varepsilon}\right\}$ is bounded in $H_{0}^{1}(\Omega)$ and if $v_{\varepsilon} \rightarrow v_{0}$ in $H_{0}^{1}(\Omega)$, we have

$$
\left.\begin{array}{rl}
-\operatorname{div}\left(A_{0} \nabla v_{0}\right) & =f \text { in } \Omega \\
v_{0} & =0 \text { on } \partial \Omega
\end{array}\right\}
$$

when $A_{\varepsilon} H$-converges to $A_{0}$ (cf. Murat [7]). We know that $\left\{v_{\varepsilon}\right\}$ does not converge strongly in $H_{0}^{1}(\Omega)$. Nevertheless, $\int_{\Omega}\left|\nabla v_{\varepsilon}\right|^{2} \mathrm{~d} x$ is bounded and hence (at least for a subsequence) converges. We would like to know if this limit can be expressed in terms of the function $v_{0}$. More generally, if $B_{\varepsilon} \in \mathcal{M}\left(\beta_{m}, \beta_{M}, \Omega\right)$, be another family of matrices, consider the 'energy' defined by

$$
\int_{\Omega} B_{\varepsilon} \nabla v_{\varepsilon} \cdot \nabla v_{\varepsilon} \mathrm{d} x
$$


Again, this is a bounded sequence and we would like to express its limit (when it converges) in terms of $v_{0}$. More precisely, we would like to know if there exists a matrix $B^{\#} \in \mathcal{M}\left(\tilde{\beta}_{m}, \tilde{\beta}_{M}, \Omega\right)$ such that

$$
\int_{\Omega} B_{\varepsilon} \nabla v_{\varepsilon} \cdot \nabla v_{\varepsilon} \mathrm{d} x \rightarrow \int_{\Omega} B^{\#} \nabla v_{0} \cdot \nabla v_{0} \mathrm{~d} x
$$

and, if so, identify that matrix and estimate the constants $\tilde{\beta}_{m}$ and $\tilde{\beta}_{M}$.

When $B_{\varepsilon}=A_{\varepsilon}$, it is well-known (cf. Murat [7]) that indeed $B^{\#}=A_{0}$, the $H$-limit of $A_{\varepsilon}$.

It turns out that the solution to this problem is closely related to the question of homogenizing an associated optimal control problem.

Let $U_{\text {ad }} \subset L^{2}(\Omega)$ be a closed convex set (called the set of admissible controls) and let $f \in L^{2}(\Omega)$ be given. Given $\theta \in U_{\text {ad }}$, let $u_{\varepsilon} \in H_{0}^{1}(\Omega)$ be the unique (weak) solution of the 'state equation':

$$
\left.\begin{array}{rlrl}
-\operatorname{div}\left(A_{\varepsilon} \nabla u_{\varepsilon}\right) & =f+\theta & & \text { in } \Omega \\
u_{\varepsilon} & =0 & & \text { on } \partial \Omega
\end{array}\right\} .
$$

Then, there exists a unique 'optimal control' $\theta_{\varepsilon}^{*} \in U_{\text {ad }}$ such that

$$
J_{\varepsilon}\left(\theta_{\varepsilon}^{*}\right)=\min _{\theta \in U_{\mathrm{ad}}} J_{\varepsilon}(\theta)
$$

where, for $\theta \in U_{\mathrm{ad}}$, and $u_{\varepsilon}=u_{\varepsilon}(\theta)$ solution of (1.3),

$$
J_{\varepsilon}(\theta)=\frac{1}{2} \int_{\Omega} B_{\varepsilon} \nabla u_{\varepsilon} \cdot \nabla u_{\varepsilon} \mathrm{d} x+\frac{N}{2} \int_{\Omega} \theta^{2} \mathrm{~d} x,
$$

$N>0$ being a fixed constant, called the 'cost of the control'.

The homogenization of the optimal control problem (1.3)-(1.5) was first studied in the periodic case by Kesavan and Vanninathan [5] and then in the geneal case under the framework of $H$-convergence by Kesavan and Saint Jean Paulin [3]. They also extended these results (cf. [4]) to the 'perforated case' where $\Omega$ is replaced by a family of 'perforated domains' $\Omega_{\varepsilon} \subset \Omega$. In all these cases, it was shown that there exists a matrix $B^{\#}$ such that, in the limit, there is an optimal control problem with $A_{0}$ and $B^{\#}$ replacing $A_{\varepsilon}$ and $B_{\varepsilon}$ respectively in (1.3)-(1.5).

The expression derived for $B^{\#}$ is complicated and the symmetry of this matrix, when all the $B_{\varepsilon}$ are symmetric, requires a detailed proof (cf. [3,4]). Further, while the ellipticity could be proved, no upper bound, i.e. an estimate for $\tilde{\beta}_{M}$, could be derived.

In this paper, a new formula for $B^{\#}$ is obtained and, in the symmetric case, the symmetry can be read off directly from it. An upper bound is also derived.

The paper is organized as follows. In $\S 2$, the equivalence of the two problems stated above is studied and the existence and uniqueness of the matrix $B^{\#}$ is established based on the results of Kesavan and Saint Jean Paulin [3]. In $\S 3$, the new formula for $B^{\#}$ is derived and its properties are studied. In $\S 4$, the corresponding results for the perforated case are stated.

\section{Two equivalent problems}

Let $\Omega \subset \mathbb{R}^{N}$ be a bounded domain and let $A_{\varepsilon} \in \mathcal{M}\left(\alpha_{m}, \alpha_{M}, \Omega\right)$ and $B_{\varepsilon} \in \mathcal{M}\left(\beta_{m}, \beta_{M}, \Omega\right)$ be given. We now consider two statements. 
(S1) There exists a matrix $B^{\#} \in \mathcal{M}\left(\tilde{\beta}_{m}, \tilde{\beta}_{M}, \Omega\right)$ such that, given a strongly convergent sequence $\left\{g_{\varepsilon}\right\}$ in $H^{-1}(\Omega)$ and the corresponding sequence $\left\{v_{\varepsilon}\right\}$ of solutions in $H_{0}^{1}(\Omega)$ of the problem

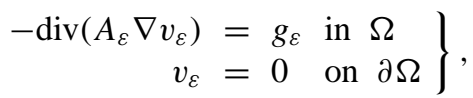

then, for a subsequence, $v_{\varepsilon} \rightarrow v_{0}$ weakly in $H_{0}^{1}(\Omega)$ and

$$
\begin{aligned}
& \int_{\Omega} B_{\varepsilon} \nabla v_{\varepsilon} \cdot \nabla v_{\varepsilon} \mathrm{d} x \rightarrow \int_{\Omega} B^{\#} \nabla v_{0} \cdot \nabla v_{0} \mathrm{~d} x, \\
& B_{\varepsilon} \nabla v_{\varepsilon} \cdot \nabla v_{\varepsilon} \rightarrow B^{\#} \nabla v_{0} \cdot \nabla v_{0} \quad \text { in } \mathcal{D}^{\prime}(\Omega) .
\end{aligned}
$$

Remark 2.1. If $g_{\varepsilon} \rightarrow g$ in $H^{-1}(\Omega)$, then (cf. Murat [7])

$$
\left.\begin{array}{rl}
-\operatorname{div}\left(A_{0} \nabla v_{0}\right)= & \text { in } \Omega \\
v_{0}= & 0 \text { on } \partial \Omega
\end{array}\right\},
$$

where $A_{0}$ is the $H$-limit of $A_{\varepsilon}$. Also

$$
A_{\varepsilon} \nabla v_{\varepsilon} \rightarrow A_{0} \nabla v_{0}
$$

weakly in $L^{2}(\Omega)^{N}$.

In order to make the second statement, we need to introduce the 'adjoint state' function. Let $g_{\varepsilon}$ and $v_{\varepsilon}$ be as above. Then we denote by $p_{\varepsilon} \in H_{0}^{1}(\Omega)$, the adjoint state, which is the solution of

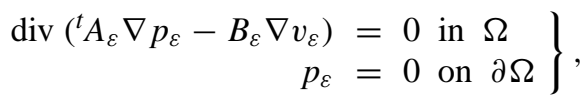

where, we have denoted the transpose of $A_{\varepsilon}$ by ${ }^{t} A_{\varepsilon}$.

Remark 2.2. From the hypotheses, it is evident that $\left\{p_{\varepsilon}\right\}$ is bounded in $H_{0}^{1}(\Omega)$.

Remark 2.3. The system consisting of (2.1) and (2.6) is of the type used by Lions [6] to construct the optimality system to solve the optimal control problem (1.3)-(1.5), using a duality argument. The system consisting of (2.1) and (2.6) was used by Kesavan and Saint Jean Paulin [3] to homogenize the optimal control problem (1.3)-(1.5).

(S2) There exists a matrix $B^{\#} \in \mathcal{M}\left(\tilde{\beta}_{m}, \tilde{\beta}_{M}, \Omega\right)$ such that, given $g_{\varepsilon}$ strongly convergent in $H^{-1}(\Omega)$ and $v_{\varepsilon}$ solution of (2.1) and $p_{\varepsilon}$ solution of (2.6), then, for a subsequence, $v_{\varepsilon} \rightarrow v_{0}, p_{\varepsilon} \rightarrow p_{0}$ weakly in $H_{0}^{1}(\Omega)$ and $z_{\varepsilon}={ }^{t} A_{\varepsilon} \nabla p_{\varepsilon}-B_{\varepsilon} \nabla v_{\varepsilon} \rightarrow z$ weakly in $L^{2}(\Omega)^{N}$, where

$$
z={ }^{t} A_{0} \nabla p_{0}-B^{\#} \nabla v_{0}
$$

and $A_{0}$ is the $H$-limit of $A_{\varepsilon}$.

Remark 2.4. The pair $\left(v_{0}, p_{0}\right)$ will satisfy the homogenized system:

$$
\left.\begin{array}{rl}
-\operatorname{div}\left(A_{0} \nabla v_{0}\right) & =g \text { in } \Omega \\
\operatorname{div}\left({ }^{t} A_{0} \nabla p_{0}-B^{\#} \nabla v_{0}\right) & =0 \text { in } \Omega \\
v_{0}=p_{0} & =0 \text { on } \partial \Omega
\end{array}\right\} .
$$

We now prove the equivalence of these two statements. 
Theorem 2.1. If $B^{\#} \in \mathcal{M}\left(\tilde{\beta}_{m}, \tilde{\beta}_{M}, \Omega\right)$ is such that (S2) is true, then it also verifies (S1). The converse is true if $B_{\varepsilon}$ and $B^{\#}$ are symmetric.

Proof. Let (S2) hold for $B^{\#}$. Now, by virtue of (2.1), (2.4), (2.6) and (2.8), we have, for the relevant subsequence,

$$
\begin{aligned}
\int_{\Omega} B_{\varepsilon} \nabla v_{\varepsilon} \cdot \nabla v_{\varepsilon} \mathrm{d} x & =\int_{\Omega}{ }^{t} A_{\varepsilon} \nabla p_{\varepsilon} \cdot \nabla v_{\varepsilon} \mathrm{d} x \\
& =\int_{\Omega} A_{\varepsilon} \nabla v_{\varepsilon} \cdot \nabla p_{\varepsilon} \mathrm{d} x \\
& =\left\langle g_{\varepsilon}, p_{\varepsilon}\right\rangle_{H^{-1}(\Omega), H_{0}^{1}(\Omega)} \\
& \rightarrow\left\langle g, p_{0}\right\rangle_{H^{-1}(\Omega), H_{0}^{1}(\Omega)} \\
& =\int_{\Omega} A_{0} \nabla v_{0} \cdot \nabla p_{0} \mathrm{~d} x \\
& =\int_{\Omega} B^{\#} \nabla v_{0} \cdot \nabla v_{0} \mathrm{~d} x .
\end{aligned}
$$

This proves (2.2). Now,

$$
B_{\varepsilon} \nabla v_{\varepsilon} \cdot v_{\varepsilon}=-\left({ }^{t} A_{\varepsilon} \nabla p_{\varepsilon}-B_{\varepsilon} \nabla v_{\varepsilon}\right) \cdot \nabla v_{\varepsilon}+\left(A_{\varepsilon} \nabla v_{\varepsilon}\right) \cdot \nabla p_{\varepsilon} .
$$

Now, by virtue of (2.1) and (2.6), the divergences of the expressions within parantheses in each of the two terms in the right-hand side are strongly convergent in $H^{-1}(\Omega)$. Also, $\nabla v_{\varepsilon}$ and $\nabla p_{\varepsilon}$ converge weakly in $L^{2}(\Omega)^{N}$. Thus, by the div-curl lemma of compensated compactness theory (cf. Murat [7], Murat and Tartar [8] or Tartar [10]), we conclude that, in view of (2.5) and (2.7),

$$
\begin{aligned}
B_{\varepsilon} \nabla v_{\varepsilon} \cdot \nabla v_{\varepsilon} & \rightarrow-\left({ }^{t} A_{0} \nabla p_{0}-B^{\#} \nabla v_{0}\right) \cdot \nabla v_{0}+A_{0} \nabla v_{0} \cdot \nabla p_{0} \\
& =B^{\#} \nabla v_{0} \cdot \nabla v_{0}
\end{aligned}
$$

in $\mathcal{D}^{\prime}(\Omega)$. This proves (2.3).

Conversely, let $B_{\varepsilon}$ and $B^{\#}$ be symmetric and assume that (S1) holds. Let $\omega \Subset \Omega$ be a (relatively compact) open subset and let $\eta \in \mathcal{D}(\Omega)$ be such that $\eta \equiv 1$ in a neighborhood of $\omega$. Define $\eta_{\varepsilon}^{k} \in H_{0}^{1}(\Omega), 1 \leq k \leq N$, to be the unique solution of the problem

$$
\left.\begin{array}{rlrl}
-\operatorname{div}\left(A_{\varepsilon} \nabla \eta_{\varepsilon}^{k}\right) & =-\operatorname{div}\left(A_{0} \nabla\left(\eta x_{k}\right)\right) & & \text { in } \Omega \\
\eta_{\varepsilon}^{k} & =0 & & \text { on } \partial \Omega
\end{array}\right\} .
$$

Then, by $H$-convergence, $\eta_{\varepsilon}^{k} \rightarrow \eta x_{k}$ weakly in $H_{0}^{1}(\Omega)$ and $A_{\varepsilon} \nabla \eta_{\varepsilon}^{k} \rightarrow A_{0} \nabla\left(\eta x_{k}\right)$ weakly in $L^{2}(\Omega)^{N}$. By superposition of the solutions of (2.1) and (2.9), we get

$$
-\operatorname{div}\left(A_{\varepsilon} \nabla\left(v_{\varepsilon} \pm \eta_{\varepsilon}^{k}\right)\right)=g_{\varepsilon} \pm\left(-\operatorname{div}\left(A_{0} \nabla\left(\eta x_{k}\right)\right) \text { in } \Omega .\right.
$$

Hence, by (S1), for a subsequence,

$$
B_{\varepsilon} \nabla\left(v_{\varepsilon} \pm \eta_{\varepsilon}^{k}\right) \cdot \nabla\left(v_{\varepsilon} \pm \eta_{\varepsilon}^{k}\right) \rightarrow B^{\#} \nabla\left(v_{0} \pm \eta x_{k}\right) \cdot \nabla\left(v_{0} \pm \eta x_{k}\right)
$$

in $\mathcal{D}^{\prime}(\Omega)$. Hence, using the polarization identity which requires the symmetry of $B_{\varepsilon}$ and $B^{\#}$, we get that

$$
B_{\varepsilon} \nabla v_{\varepsilon} \cdot \nabla \eta_{\varepsilon}^{k} \rightarrow B^{\#} \nabla v_{0} \cdot \nabla\left(\eta x_{k}\right)
$$


in $\mathcal{D}^{\prime}(\Omega)$. We can now apply the div-curl lemma to the pair $\left(z_{\varepsilon}, \nabla \eta_{\varepsilon}^{k}\right)$, since div $z_{\varepsilon}=0$, to get

$$
z_{\varepsilon} \cdot \nabla \eta_{\varepsilon}^{k} \rightarrow z \cdot \nabla\left(\eta x_{k}\right)
$$

in $\mathcal{D}^{\prime}(\Omega)$. On the other hand

$$
\begin{aligned}
z_{\varepsilon} \cdot \nabla \eta_{\varepsilon}^{k} & =A_{\varepsilon} \nabla \eta_{\varepsilon}^{k} \cdot \nabla p_{\varepsilon}-B_{\varepsilon} \nabla v_{\varepsilon} \cdot \nabla \eta_{\varepsilon}^{k} \\
& \rightarrow A_{0} \nabla\left(\eta x_{k}\right) \cdot \nabla p_{0}-B^{\#} \nabla v_{0} \cdot \nabla\left(\eta x_{k}\right)
\end{aligned}
$$

again by applying the div-curl lemma to the first term on the right-hand side and by also using (2.10). Thus, on $\omega$, we have

$$
z \cdot e_{k}={ }^{t} A_{0} \nabla p_{0} \cdot e_{k}-B^{\#} \nabla v_{0} \cdot e_{k}
$$

where $e_{k}$ is the standard $k$-th basis vector of $\mathbb{R}^{N}$. This proves (2.7) on $\omega$ and as $\omega \Subset \Omega$ was arbitrary, we have the result on $\Omega$.

Remark 2.5. We can use the test functions $\eta_{\varepsilon}^{j}$ to prove the uniqueness of the matrix $B^{\# \text {, }}$ when it exists. Indeed, if we have two matrices $B_{i}^{\#}, i=1,2$, satisfying (S2), (or (S1), in the symmetric case), setting $v_{\varepsilon}=\eta_{\varepsilon}^{j}$, we then have that (cf. (2.7))

$$
{ }^{t} A_{0} \nabla p_{0}-B_{1}^{\#} \nabla\left(\eta x_{j}\right)={ }^{t} A_{0} \nabla p_{0}-B_{2}^{\#} \nabla\left(\eta x_{j}\right) .
$$

Thus on $\omega$, we have that $B_{1}^{\#}=B_{2}^{\#}$ and the result follows for all of $\Omega$ since $\omega \Subset \Omega$ is arbitrary.

The existence of a $B^{\#}$ satisfying (S2) was proved in the general case by Kesavan and Saint Jean Paulin [3]. We recall their formulation and also give another, shorter, proof of their result.

We first need to define some test functions. First of all, we recall the existence of functions $X_{\varepsilon}^{k} \in H^{1}(\Omega)$, for $1 \leq k \leq N$, with the following properties (cf. Murat [7]).

$$
\left.\begin{array}{c}
X_{\varepsilon}^{k} \rightarrow x_{k} \text { weakly in } H^{1}(\Omega) \\
A_{\varepsilon} \nabla X_{\varepsilon}^{k} \rightarrow A_{0} e_{k} \text { weakly in } L^{2}(\Omega)^{N} \\
\left.\mathrm{v}\left(A_{\varepsilon} \nabla X_{\varepsilon}^{k}\right)\right\} \text { converges strongly in } H^{-1}(\Omega)
\end{array}\right\} .
$$

We now define another set of test functions $\psi_{\varepsilon}^{k} \in H_{0}^{1}(\Omega)$ for $1 \leq k \leq N$, which verify

$$
\left.\begin{array}{rl}
-\operatorname{div}\left({ }^{t} A_{\varepsilon} \nabla \psi_{\varepsilon}^{k}+{ }^{t} B_{\varepsilon} \nabla X_{\varepsilon}^{k}\right) & =0 \text { in } \Omega \\
\psi_{\varepsilon}^{k} & =0 \text { on } \partial \Omega
\end{array}\right\} .
$$

Then, up to a subsequence, $\left\{\psi_{\varepsilon}^{k}\right\}$ converges weakly in $H_{0}^{1}(\Omega)$ to $\psi_{0}^{k}$ and $\left\{{ }^{t} A_{\varepsilon} \nabla \psi_{\varepsilon}^{k}+\right.$ $\left.{ }^{t} B_{\varepsilon} \nabla X_{\varepsilon}^{k}\right\}$ converges weakly in $L^{2}(\Omega)^{N}$. Then, (cf. [3]), we define

$$
{ }^{t}\left(B^{\#}\right) e_{k}=\lim _{\varepsilon \rightarrow 0}\left({ }^{t} A_{\varepsilon} \nabla \psi_{\varepsilon}^{k}+{ }^{t} B_{\varepsilon} \nabla X_{\varepsilon}^{k}\right)-{ }^{t} A_{0} \nabla \psi_{0}^{k} .
$$

Theorem 2.2. $B^{\#}$ defined by (2.13) satisfies (S2). 
Proof. We extract a subsequence such that all the bounded sequences that occur below are convergent in the relevant weak topologies. Let $g_{\varepsilon} \rightarrow g$ strongly in $H^{-1}(\Omega)$ and $\left(v_{\varepsilon}, p_{\varepsilon}\right) \in H_{0}^{1}(\Omega) \times H_{0}^{1}(\Omega)$ be the solution of (2.1) and (2.6). Let $v_{\varepsilon} \rightarrow v_{0}$ and $p_{\varepsilon} \rightarrow p_{0}$ weakly in $H_{0}^{1}(\Omega)$ and let $z_{\varepsilon} \rightarrow z$ weakly in $L^{2}(\Omega)^{N}$. Now

$$
\begin{aligned}
z_{\varepsilon} \cdot \nabla X_{\varepsilon}^{k}= & \nabla p_{\varepsilon} \cdot A_{\varepsilon} \nabla X_{\varepsilon}^{k}-{ }^{t} B_{\varepsilon} \nabla X_{\varepsilon}^{k} \cdot \nabla v_{\varepsilon} \\
= & \left(A_{\varepsilon} \nabla X_{\varepsilon}^{k}\right) \cdot \nabla p_{\varepsilon}-\left({ }^{t} A_{\varepsilon} \nabla \psi_{\varepsilon}^{k}+{ }^{t} B_{\varepsilon} \nabla X_{\varepsilon}^{k}\right) \cdot \nabla v_{\varepsilon} \\
& +\left(A_{\varepsilon} \nabla v_{\varepsilon}\right) \cdot \nabla \psi_{\varepsilon}^{k}
\end{aligned}
$$

We can pass to the limit, using the div-curl lemma, in each term to get

$$
z \cdot e_{k}=A_{0} e_{k} \cdot \nabla p_{0}-\lim _{\varepsilon \rightarrow 0}\left({ }^{t} A_{\varepsilon} \nabla \psi_{\varepsilon}^{k}+{ }^{t} B_{\varepsilon} \nabla X_{\varepsilon}^{k}\right) \cdot \nabla v_{0}+A_{0} \nabla v_{0} \cdot \nabla \psi_{0}^{k}
$$

using (2.13) from which (2.7) follows.

Remark 2.6. In the statements (S1) and (S2), we have required that the relevant convergences occur for a subsequence. It must be noted that the subsequence is independent of the strongly convergent sequence $g_{\varepsilon}$. Indeed, it depends only on the convergences implied in (2.11) and (2.13) (cf. Rajesh [9]).

\section{Properties of $B^{\#}$}

We are now interested in properties like the symmetry and ellipticity of the matrix $B^{\#}$ defined in the previous section. Kesavan and Saint Jean Paulin [3] proved that it is symmetric when all the $B_{\varepsilon}$ are symmetric and that $\tilde{\beta}_{m}=\beta_{m}$. However, the problem of estimating $\tilde{\beta}_{M}$ was left open. In this section, a new formula for $B^{\#}$ will be given from which the symmetry can be just read off and which will also enable us to estimate $\tilde{\beta}_{M}$.

First of all, we recall the corrector matrices occurring in the study of $H$-convergence of $A_{\varepsilon}$, as introduced by Murat [7]. If $X_{\varepsilon}^{k}$ are the test functions introduced in the previous section (cf. (2.11)), the corrector matrices are defined by

$$
M_{\varepsilon} e_{k}=\nabla X_{\varepsilon}^{k}, \quad 1 \leq k \leq N .
$$

Then, the following properties hold (cf. Murat [7] or Murat and Tartar [8]):

$$
\left.\begin{array}{c}
M_{\varepsilon} \rightarrow I \text { weakly in } L^{2}(\Omega)^{N^{2}} \\
A_{\varepsilon} M_{\varepsilon} \rightarrow A_{0} \text { weakly in } L^{2}(\Omega)^{N^{2}} \\
{ }^{t} M_{\varepsilon} A_{\varepsilon} M_{\varepsilon} \rightarrow A_{0} \text { in } \mathcal{D}^{\prime}(\Omega)^{N^{2}} \\
\left\{\operatorname{div}\left(A_{\varepsilon} M_{\varepsilon}\right)\right\} \text { converges strongly in } H^{-1}(\Omega)^{N}
\end{array}\right\}
$$

We now prove the main result of this section.

Theorem 3.1. $B^{\#}$ defined by (2.13) is the limit, in the sense of distributions, of ${ }^{t} M_{\varepsilon} B_{\varepsilon} M_{\varepsilon}$.

Proof. It is enough to show that, for $1 \leq i, j \leq N$,

$$
{ }^{t} M_{\varepsilon} B_{\varepsilon} M_{\varepsilon} e_{k} \cdot e_{j} \rightarrow B^{\#} e_{k} \cdot e_{j}
$$


in $\mathcal{D}^{\prime}(\Omega)$. Now

$$
\begin{aligned}
{ }^{t} M_{\varepsilon} B_{\varepsilon} M_{\varepsilon} e_{k} \cdot e_{j} & ={ }^{t} B_{\varepsilon} M_{\varepsilon} e_{j} \cdot M_{\varepsilon} e_{k} \\
& =\left({ }^{t} A_{\varepsilon} \nabla \psi \psi_{\varepsilon}^{j}+{ }^{t} B_{\varepsilon} M_{\varepsilon} e_{j}\right) \cdot M_{\varepsilon} e_{k}-{ }^{t} A_{\varepsilon} \nabla \psi \psi_{\varepsilon}^{j} \cdot M_{\varepsilon} e_{k} \\
& =\left({ }^{t} A_{\varepsilon} \nabla \psi \psi_{\varepsilon}^{j}+{ }^{t} B_{\varepsilon} \nabla X_{\varepsilon}^{j}\right) \cdot \nabla X_{\varepsilon}^{k}-A_{\varepsilon} \nabla X_{\varepsilon}^{k} \cdot \nabla \psi_{\varepsilon}^{j} .
\end{aligned}
$$

We can pass to the limit in each of the two terms on the right-hand side using the div-curl lemma to get

$$
{ }^{t} M_{\varepsilon} B_{\varepsilon} M_{\varepsilon} e_{k} \cdot e_{j} \rightarrow{ }^{t} B^{\#} e_{j} \cdot e_{k}=B^{\#} e_{k} \cdot e_{j}
$$

\section{COROLLARY 3.1}

If the $B_{\varepsilon}$ are symmetric, then so is $B^{\#}$.

Theorem 3.2. $B^{\#} \in \mathcal{M}\left(\tilde{\beta}_{m}, \tilde{\beta}_{M}, \Omega\right)$, where $\tilde{\beta}_{m}=\beta_{m}$ and

$$
\tilde{\beta}_{M}=\beta_{M}\left(\frac{\alpha_{M}}{\alpha_{m}}\right)^{2}
$$

Proof. That $\tilde{\beta}_{m}=\beta_{m}$ has already been proved in [3]. Let $\varphi \in \mathcal{D}(\Omega), \varphi \geq 0$ and let $\xi \in \mathbb{R}^{N}$. Then

$$
\begin{aligned}
\int_{\Omega} B_{\varepsilon} M_{\varepsilon} \xi \cdot M_{\varepsilon} \xi \varphi \mathrm{d} x & \leq B_{M} \int_{\Omega}\left|M_{\varepsilon} \xi\right|^{2} \varphi \mathrm{d} x \\
& \leq \frac{\beta_{M}}{\alpha_{m}} \int_{\Omega} A_{\varepsilon} M_{\varepsilon} \xi \cdot M_{\varepsilon} \xi \varphi \mathrm{d} x \\
& =\frac{\beta_{M}}{\alpha_{m}} \int_{G}{ }^{t} M_{\varepsilon} A_{\varepsilon} M_{\varepsilon} \xi \cdot \xi \varphi \mathrm{d} x
\end{aligned}
$$

Passing to the limit, using Theorem 3.1 and (3.2), we get

$$
\int_{\Omega} B^{\#} \xi \cdot \xi \varphi \mathrm{d} x \leq \frac{\beta_{M}}{\alpha_{m}} \int_{\Omega} A_{0} \xi \cdot \xi \varphi \mathrm{d} x \leq \frac{\beta_{M}}{\alpha_{m}} \frac{\alpha_{M}^{2}}{\alpha_{m}}|\xi|^{2} \int_{\Omega} \varphi \mathrm{d} x,
$$

since we know that $A_{0} \in \mathcal{M}\left(\tilde{\alpha}_{m}, \tilde{\alpha}_{M}, \Omega\right)$, where $\tilde{\alpha}_{m}=\alpha_{m}$ and $\tilde{\alpha}_{M}=\alpha_{M}^{2} / \alpha_{m}$ (cf. Murat [7]). Since $\varphi$ was arbitrary, this proves the theorem.

Remark 3.1. Consider the one-dimensional case. Let $0<\alpha_{M} \leq a_{\varepsilon}(x) \leq \alpha_{M}$ and $0<\beta_{M}$ $\leq b_{\varepsilon}(x) \leq \beta_{M}$. Let

$$
\begin{array}{rlrl}
-\frac{d}{d x}\left(a_{\varepsilon} \frac{d u_{\varepsilon}}{d x}\right) & =f & \text { in }(0,1) \\
u_{\varepsilon}(0) & =u_{\varepsilon}(1)=0 .
\end{array}
$$

Then, it has been shown by Kesavan and Saint Jean Paulin [3] that

$$
\int_{0}^{1} b_{\varepsilon} \frac{\mathrm{d} u_{\varepsilon}}{\mathrm{d} x} \frac{\mathrm{d} u_{\varepsilon}}{\mathrm{d} x} \mathrm{~d} x \rightarrow \int_{0}^{1} b^{\#} \frac{\mathrm{d} u_{0}}{\mathrm{~d} x} \frac{\mathrm{d} u_{0}}{\mathrm{~d} x} \mathrm{~d} x
$$


with

$$
b^{\#}=\frac{a_{0}^{2}}{g_{0}},
$$

where

$$
\frac{1}{a_{\varepsilon}} \rightarrow \frac{1}{a_{0}} \quad \text { and } \quad \frac{1}{g_{\varepsilon}} \equiv \frac{b_{\varepsilon}}{a_{\varepsilon}^{2}}-\frac{1}{g_{0}} \quad \text { in } \quad L^{\infty}(0,1) \text { weak -*. }
$$

This yields precisely the bound obtained above for $b^{\#}$.

\section{The perforated case}

We now briefly describe the problem in the perforated case and state the results without proofs, since those of the corresponding results in the previous case carry over mutatis mutandis.

Let $\Omega \subset \mathbb{R}^{N}$ be a bounded domain and for $\varepsilon>0$, let $S_{\varepsilon} \subset \Omega$ be a closed set (the set of perforations). We call $\Omega_{\varepsilon}=\Omega \backslash S_{\varepsilon}$ the perforated domain. Following Briane, Damlamian and Donato [1], we say that a family $\left\{S_{\varepsilon}\right\}$ of holes is admissible if the following conditions are fulfilled.

H1 If $\chi_{\varepsilon}$ is the characteristic function of $S_{\varepsilon}$, then every weak-* limit of $\left\{\chi_{\varepsilon}\right\}$ in $L^{\infty}(\Omega)$ is positive a.e.

H2 Let

$$
V_{\varepsilon}=\left\{u \in H^{1}\left(\Omega_{\varepsilon}\right) \mid u=0 \text { on } \partial \Omega\right\} .
$$

Then, there exists an extension operator $P_{\varepsilon}: V_{\varepsilon} \rightarrow H_{0}^{1}(\Omega)$ and a constant $C_{0}>0$, independent of $\varepsilon$ such that, for any $u \in V_{\varepsilon}$,

$$
\left.P_{\varepsilon} u\right|_{\Omega_{\varepsilon}}=u \quad \text { and } \quad\left\|\nabla P_{\varepsilon} u\right\|_{L^{2}(\Omega)^{N}} \leq C_{0}\|\nabla u\|_{L^{2}\left(\Omega_{\varepsilon}\right)^{N}} .
$$

Analogous to the theory of $H$-convergence, we have a theory of $H_{0}$-convergence (cf. [1]).

We now assume that we have two families of matrices $A_{\varepsilon} \in \mathcal{M}\left(\alpha_{m}, \alpha_{M}, \Omega\right)$ and $B_{\varepsilon} \in$ $\mathcal{M}\left(\beta_{m}, \beta_{M}, \Omega\right)$. We denote by $P_{\varepsilon}^{*}: H^{-1}(\Omega) \rightarrow V_{\varepsilon}^{*}$, the adjoint of $P_{\varepsilon}$. For functions in $L^{2}\left(\Omega_{\varepsilon}\right)$, we also have the trivial extension operator $Q_{\varepsilon}$ which extends the functions by zero across the holes to give a function in $L^{2}(\Omega)$. We denote the unit outward normal (with respect to $\Omega_{\varepsilon}$ ) on $\partial S_{\varepsilon}$ be $n_{\varepsilon}$.

(S3) There exists a matrix $B^{\#} \in \mathcal{M}\left(\tilde{\beta}_{m}, \tilde{\beta}_{M}, \Omega\right)$ such that, given a strongly convergent sequence $\left\{g_{\varepsilon}\right\}$ in $H^{-1}(\Omega)$ and the corresponding sequence of solutions $v_{\varepsilon} \in V_{\varepsilon}$ of

$$
\left.\begin{array}{rlrl}
-\operatorname{div}\left(A_{\varepsilon} \nabla v_{\varepsilon}\right) & =P_{\varepsilon}^{*} g_{\varepsilon} & & \text { in } \Omega_{\varepsilon} \\
A_{\varepsilon} \nabla v_{\varepsilon} \cdot n_{\varepsilon} & =0 & & \text { on } \partial S_{\varepsilon} \\
v_{\varepsilon} & =0 & & \text { on } \partial \Omega
\end{array}\right\},
$$

then, for a subsequence, $P_{\varepsilon} v_{\varepsilon} \rightarrow v_{0}$ weakly in $H_{0}^{1}(\Omega)$ and

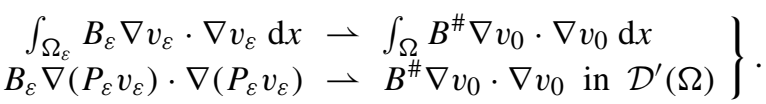

(S4) There exists a matrix $B^{\#} \in \mathcal{M}\left(\tilde{\beta}_{m}, \tilde{\beta}_{M}, \Omega\right)$ such that given a strongly convergent sequence $\left\{g_{\varepsilon}\right\}$ in $H^{-1}(\Omega)$ and the sequence $\left\{v_{\varepsilon}\right\}$ of solutions of (4.2) and the sequence $\left\{p_{\varepsilon}\right\}$ in $V_{\varepsilon}$ of solutions of 


$$
\begin{aligned}
& \operatorname{div}\left({ }^{t} A_{\varepsilon} \nabla p_{\varepsilon}-B_{\varepsilon} \nabla v_{\varepsilon}\right)=0 \text { in } \Omega_{\varepsilon} \\
& \left.\left({ }^{t} A_{\varepsilon} \nabla p_{\varepsilon}-B_{\varepsilon} \nabla v_{\varepsilon}\right) \cdot n_{\varepsilon}=0 \text { on } \partial S_{\varepsilon}\right\} \text {, } \\
& p_{\varepsilon}=0 \text { on } \partial \Omega
\end{aligned}
$$

then, for a subsequence, $P_{\varepsilon} v_{\varepsilon} \rightarrow v_{0}, P_{\varepsilon} p_{\varepsilon} \rightarrow p_{0}$ weakly in $H_{0}^{1}(\Omega), z_{\varepsilon}=Q_{\varepsilon}\left({ }^{t} A_{\varepsilon} \nabla p_{\varepsilon}-\right.$ $\left.B_{\varepsilon} \nabla v_{\varepsilon}\right) \rightarrow z$ weakly in $L^{2}(\Omega)^{N}$ where

$$
z={ }^{t} A_{0} \nabla p_{0}-B^{\#} \nabla v_{0}
$$

$A_{0}$ being the $H_{0}$-limit of $\left\{A_{\varepsilon}\right\}$.

Theorem 4.1. If $B^{\#} \in \mathcal{M}\left(\tilde{\beta}_{m}, \tilde{\beta}_{M}, \Omega\right)$ is such that (S4) is true, then so is (S3). The converse holds when the $B_{\varepsilon}$ and $B^{\#}$ are symmetric.

Kesavan and Saint Jean Paulin [4] gave a formula for a matrix $B^{\#}$ such that (S4) holds. If we define the corrector matrices $\tilde{M}_{\varepsilon}$ by

$$
\tilde{M}_{\varepsilon} e_{k}=\nabla\left(P_{\varepsilon} \tilde{X}_{\varepsilon}^{k}\right)
$$

where the $\tilde{X}_{\varepsilon}^{k}$ are test functions with properties analogous to those mentioned in (2.11) (cf. [1] or [4]), it can be shown that (cf. Rajesh [9])

$$
\chi_{\varepsilon}{ }^{t} \tilde{M}_{\varepsilon} B_{\varepsilon} \tilde{M}_{\varepsilon} \rightarrow B^{\#}
$$

in the sense of distributions. Thus, if $B_{\varepsilon}$ are symmetric, so is $B^{\#}$ and we can show that

$$
\tilde{\beta}_{M}=\beta_{M}\left(\frac{\alpha_{M}}{\alpha_{m}}\right)^{2}
$$

Kesavan and Saint Jean Paulin [4] proved that for all $w \in H_{0}^{1}(\Omega)$

$$
\int_{\Omega} B^{\#} \nabla w \cdot \nabla w \mathrm{~d} x \geq \beta_{m} C_{0}^{-2}\|w\|_{H_{0}^{1}(\Omega)}^{2}
$$

This, in fact, implies that $\tilde{\beta}_{m}=\beta_{m} C_{0}^{-2}$. We give a proof of this below. It is adapted from a similar proof by Casado-Díaz [2], but with different test functions.

Lemma 4.1. Let $A=A(x)$ be a symmetric $N \times N$ matrix with coefficients in $L^{\infty}(\Omega)$ such that

$$
\int_{\Omega} A \nabla w \cdot \nabla w \mathrm{~d} x \geq 0
$$

for all $w \in H_{0}^{1}(\Omega)$. Then

$$
A(x) \xi \cdot \xi \geq 0
$$

a.e. in $\Omega$ for all $\xi \in \mathbb{R}^{N}$. 
Proof. Let $\xi \in \mathbb{R}^{N}$ and let $\varphi \geq 0$ be a $C_{0}^{1}$-function. Define

$$
\begin{aligned}
v_{\varepsilon}(x) & =\varepsilon \cos \left(\varepsilon^{-1} \xi \cdot x\right) \varphi(x), \\
w_{\varepsilon}(x) & =\varepsilon \sin \left(\varepsilon^{-1} \xi \cdot x\right) \varphi(x) .
\end{aligned}
$$

Then $v_{\varepsilon}, w_{\varepsilon} \in H_{0}^{1}(\Omega)$. Applying (4.6) to both $v_{\varepsilon}$ and $w_{\varepsilon}$ and adding the resulting inequalities, we get

$$
\varepsilon^{2} \int_{\Omega} A \nabla \varphi \cdot \nabla \varphi \mathrm{d} x+\int_{\Omega}(A \xi \cdot \xi) \varphi^{2} \mathrm{~d} x \geq 0 .
$$

Passing to the limit as $\varepsilon \rightarrow 0$, using the arbitrariness of $\varphi$, we get (4.7) by standard arguments.

\section{References}

[1] Briane M, Damlamian A and Donato P, $H$-convergence for perforated domains, in: Nonlinear Partial Differential Equations and their Applications, Collège de France Seminar, Vol. XIII, Pitman Research Notes in Mathematics, 1996

[2] Casado-Díaz J, Personal Communication

[3] Kesavan S and Saint Jean Paulin J, Homogenization of an optimal control problem, SIAM J. Control Optim. 35 (1997) 1557-1573

[4] Kesavan S and Saint Jean Paulin J, Optimal control on perforated domains, J. Math. Anal. Appl. 229 (1999) 563-586

[5] Kesavan S and Vanninathan M, L'homogénéisation d'un problème de contrôle optimal, C. R. Acad. Sci., Paris, Série A, 285 (1977) 441-444

[6] Lions J L, Optimal Control of Systems Governed by Partial Differential Equations (Berlin: Springer-Verlag) (1971)

[7] Murat F, $H$-convergence, Mimeographed notes, Séminaire d'Analyse Fonctionnelle et Numérique, Université d'Alger, 1977/78

[8] Murat F and Tartar L, $H$-convergence, in: Topics in the Mathematical Modelling of Composite Materials (eds) A Cherkaev and R Kohn (Birkhauser) (1997) 21-43

[9] Rajesh M, Some Problems in Homogenization, Thesis (Indian Statistical Institute, Calcutta) (2000)

[10] Tartar L, Compensated compactness and applications to partial differential equations, in: Nonlinear Analysis and Mechanics, Heriott Watt Symposium (ed) R J Knops, Pitman Research Notes in Mathematics, 39 (1979) 136-212. 